TABLE VIII.-Causes of Deaths*

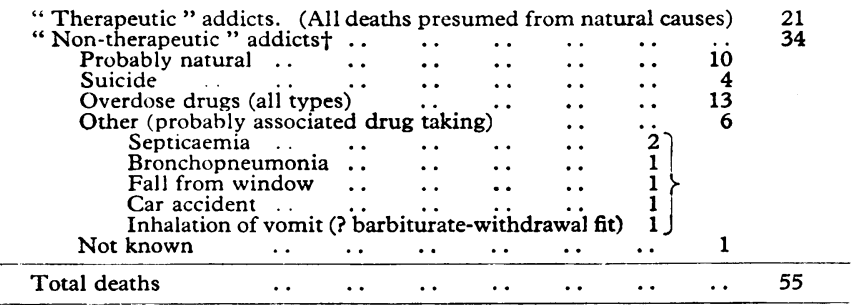

* Includes 13 subsequent deaths.

+ Mean age of "non-therapeutic" addicts at death 34 years.

(2) A large group of young British addicts, rapidly increasing, whose members have acquired their addiction from some non-therapeutic source.

(3) A group of older addicts who came to this country after the rise in numbers was first noted. The mean age of this group is 10 years higher than that of the British addicts. Most have been addicted for many years, having become addicted from a non-therapeutic source.

Addicts who have become addicted from a non-therapeutic source act as a further source of spread of addiction (Bewley, 1965). Those addicted in the course of treatment do not do this. The probable reason is that the former group contain a greater number of addicts with deviant personalities and with less satisfactory backgrounds (Chien, 1964).

\section{Conclusions}

The results of treatment of this type of addiction are very poor. Retterstol and Sund (1964) showed that despite careful treatment of drug abusers with a better prognosis, $60 \%$ continued to misuse drugs. Where poorly motivated addicts have been treated, only $10 \%$ to $30 \%$ may give up drugs (Hunt and Odoroff, 1962 ; Duvall et al., 1963). In view of this very poor response to treatment prevention is important. The practice of prescribing drugs for maintenance of addicts worked successfully only when the majority of addicts become addicted from treatment. Treating other addicts this way makes it easier for new individuals to become addicted to these drugs. Liberal prescribing has no beneficial effect on the addict (Glatt, 1965).
The chief source of illicit heroin and cocaine in this country is the sale of these drugs by addicts who have more than they' need prescribed for them (Howard, 1965 ; James, 1965). It is essential to limit the prescribing of heroin for addicts. The argument that this might lead to the creation of a black market is irrelevant, as the present practice supplies drugs free for illicit sale and encourages spread of further addiction. Addiction of this type with epidemic case-to-case spread is a Public Health problem and should be recognized as such. Statutory notification of addiction and case finding among contacts are necessary. More up-to-date information should be available. As previously suggested, a special treatment unit or treatment units and continuous review of the problem would also be desirable (Lancet, 1965).

\section{Summary}

A survey and follow-up is presented of the 507 recorded heroin addicts in the United Kingdom between 1954 and 1964. The rate of appearance of new cases has steadily increased. Over $80 \%$ of all addicts remained addicted or died. Present methods of dealing with this addiction are ineffectual. Preventive measures are recommended, including limitations on prescribing heroin for addicts.

I would like to thank the Home Office for giving me every facility to carry out this survey and particularly Mr. H. B. Spear, whose invaluable help has made it possible.

\section{REFERENCES}

Bewley, T. (1965). Lancet, 1, 808.

Chein, I., Gerard, D. L., Lee, R. S., and Rosenfeld, E. (1964). Narcotics Delinquency and Social Policy. Basic Books, New York, U.S.A.

Duvall, H. J., Locke, B. Z., and Brill, L. (1963). Publ. Hith Rep. (W ash.), 78, 185.

Glatt, M. M. (1965). Lancet, 2, 171.

Howard, A. (1965). Med.-leg. f. (Camb.), 33(2), 56.

Hunt, G. H., and Odoroff, M. E. (1962). Publ. Hith Rep. (Wash.), 77,

Isbell, H., Altschul, S., Kornetsky, C. H., Eisenman, A. J., Flanavy, H. G., and Fraser, H. F. (1950). Arch. Neurol. Psychiat. (Chic.), 64, 1 .

James, I. P. (1965). Lancet, 2, 288.

Lancet, 1964, 1, 649.

Lancet 1965, 2, 169.

Reports to the United Nations on the Working of the International Treaties on Narcotic Drugs. Home Office, London (1954-1964). Retterstol, N., and Sund, H. (1964). Acta psychiat. scand., 40, Suppl.
No. 179.

\title{
Laboratory Diagnosis of Infections of the Urinary Tract in General Practice by Means of a Dip-inoculum Transport Medium
}

\author{
J. P. MACKEY,* M.B., F.C.PATH., D.P.H., D.T.M.\&H., DIP.BACT. ; G. H. SANDYS,* F.I.M.L.T.
}

Brit. med. F., 1965, 2, 1286-1288

The frequency and importance of infections of the urinary tract are now generally recognized, especially in females and particularly in pregnancy, and the possible association with the later development of chronic pyelonephritis (Kass, 1960 ; Brumfitt and Percival, 1964).

Most of these infections may be diagnosed and treated in the patients' homes by the general practitioner (Mond, Percival, Williams, and Brumfitt, 1965 ; Gallagher, Montgomerie, and North, 1965) if bacteriological facilities are available.

The laboratory diagnosis requires an assessment of the bacterial concentration in the freshly passed "clean" midstream specimen of urine in order that one can distinguish the

* Public Health Laboratory, Bath. "significant" bacteriuria of infection (usually greater than 100,000 bacteria per $\mathrm{ml}$. of urine) from the relatively low bacterial concentration (under $10,000 / \mathrm{ml}$.) associated with the presence of contaminant bacteria only (Kass, 1956).

The most satisfactory way of assessing the bacterial concentration in a sample of urine is by a viable count, for which a number of techniques are available. All of them, however, depend on the rapid transmission of the specimen to the laboratory, and refrigerator storage if there is a delay before dispatch. In our experience the average general practitioner is seldom able to deliver the sample of urine to the laboratory quickly enough. Even if this could be done he must often want to take the specimen before prescribing treatment, at times when the laboratory is closed except for emergency work. For these and other reasons the practice of submitting samples of urine 
by post has been allowed to continue even though such specimens are useless for viable count by the time they reach the laboratory.

Clearly there is an urgent need for a simple transport-medium outfit capable of inoculation (by relatively untrained personnel) with a standard quantity of "clean" freshly passed mid-stream urine before its dispatch to the laboratory. In devising such an outfit it is necessary to ensure that the technique of inoculation is rapid, very simple, and reliable. The inoculated medium must be capable of inhibiting spread by Proteus sp., and after reaching the laboratory, if necessary after 24-48 hours' delay through the post, be capable of demonstrating the identity and concentration of the bacteria originally present in the fresh inoculum.

The blotting-paper inoculum technique (Leigh and Williams, 1964) was adapted and tried with some success, but was later replaced by the dip-inoculum transport medium (D.I.T.M.) described below.

\section{Materials and Methods}

The D.I.T.M. outfit consists of a 1-oz. (28-ml.) universal screw-capped bottle containing a small amount of absorbent cotton-wool at the bottom and a spoon holding a suitable medium.

Preparation of Screw-capped Bottles.-Into a 1-oz. (28-ml.) universal screw-capped bottle is placed a small quantity of cotton-wool. To the bottle is added $2 \mathrm{ml}$. of distilled water,

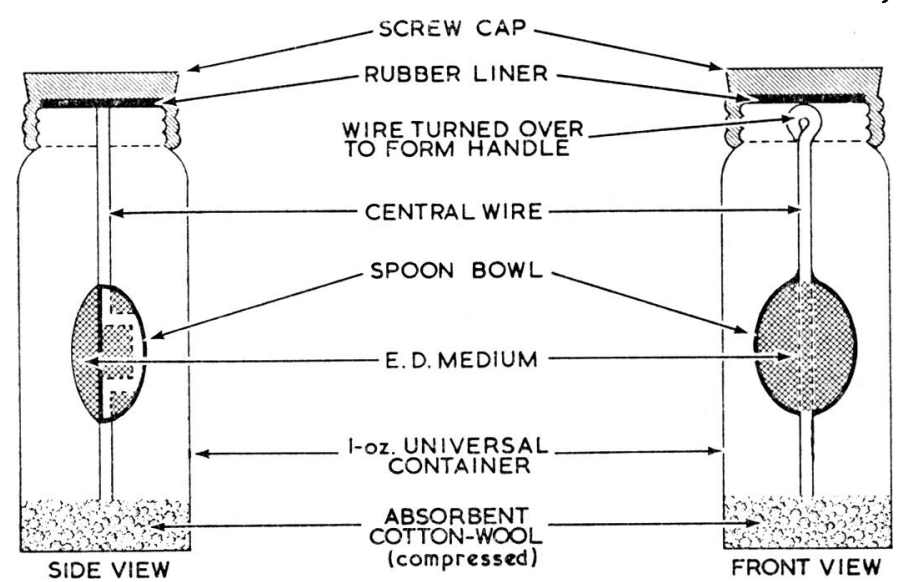

FIG. 1.-Dip-inoculum transport-medium outfit.

and the damp cotton-wool is pressed down with a glass rod to form a level pad $1 \mathrm{~cm}$. deep. The bottles are then sterilized by autoclaving at $10 \mathrm{lb}$. $(0.7 \mathrm{~kg} . / 59 \mathrm{~cm}$.) for 20 minutes.

Design of Media Spoons.-Ideally the spoons should be made of disposable sterile plastic, as used for disposable Petri dishes. For the purpose of this investigation, however, the spoons were made of non-ferrous metal and were nickelplated. ${ }^{1}$ Before use, in order to prevent contact between the metal and medium and thus avoid formation of antibacterial salts associated with heavy metals, it was found necessary to coat the spoons with polyurethane. ${ }^{2}$ This coating stands repeated boiling satisfactorily, though a slight yellowing may occur. Should the coating become damaged it can easily be replaced by removing the old coat in chloroform and recoating with fresh polyurethane. The spoon (Fig. 1) consists of an oval bowl 23 by $18 \mathrm{~mm}$. soldered to a central wire. The wire inside the bowl forms a " $U$ " shape, which in use prevents the medium from becoming detached from the bowl. The central wire extends above the bowl for $38 \mathrm{~mm}$., ending in a small

\footnotetext{
1 The metal spoons are manufactured by the Medical Wire and Equipment Co. (Bath) Ltd., Potley, Corsham, Wilts.

2 The polyurethane used is in the form of clear polyurethane paint which dries in one to two hours. It is manufactured by A. Sanderson and Co. Ltd., Hull, under the trade name Translac, and is obtainable
} from branches of the Halford Cycle Co. Ltd. loop, and extends below the bowl for $15 \mathrm{~mm}$. When the spoon is introduced into the bottle, and the bottom wire is put into the centre of the cotton-wool and the screw-cap replaced, the bowl remains in the centre of the bottle without touching the sides.

Medium for D.I.T.M.-The necessary criteria for a suitable medium are that it must be non-inhibitory, prevent the swarming of Proteus sp., and facilitate good colonial recognition and differentiation. E.D. medium (Sandys, 1960), modified for use in urine bacteriology by replacing the mannitol with $1 \%$ lactose and $0.2 \%$ sucrose and increasing the indicator strength to $0.002 \%$ and the agar strength to $1.5 \%$, has been found to be very suitable and has been used throughout this investigation.

Preparation of Media Spoons.-The media spoons are sterilized. Owing to the polyurethane coating this has to be done by boiling in distilled water. A suitable rack for holding the spoons during the addition of the medium is required. The agar medium to be used is melted and then cooled to $50^{\circ} \mathrm{C}$. The molten agar is run into the bowls carefully by means of a $10-\mathrm{ml}$. pipette, each bowl holding approximately $1.75 \mathrm{ml}$. of medium. The agar is added until it wells up above the level of the bowl rim without overflowing. The technique is carried out aseptically, and as soon as the agar is set the spoons are introduced into the prepared screw-capped bottles. The completed outfits are incubated first at $37^{\circ} \mathrm{C}$. overnight and then at room temperature for 72 hours as a confirmation of their sterility. For repeated use the agar is removed from the spoon with a wooden applicator and the spoon is resterilized in the manner described.

Instructions for Use.-For use the cap of the bottle is removed. The loop at the end of the central wire is grasped between the finger and thumb, and the spoon removed from its bottle. The medium spoon is dipped into the specimen of fresh "clean" mid-stream urine so as to just immerse the medium. The spoon is then returned to its bottle, taking care to place the bottom wire in the centre of the cotton-wool pad. It is kept in the vertical position for one minute in order to allow drainage of excess fluid from the spoon into the cottonwool via the central wire. The bottle is finally sealed by firmly replacing the screw cap.

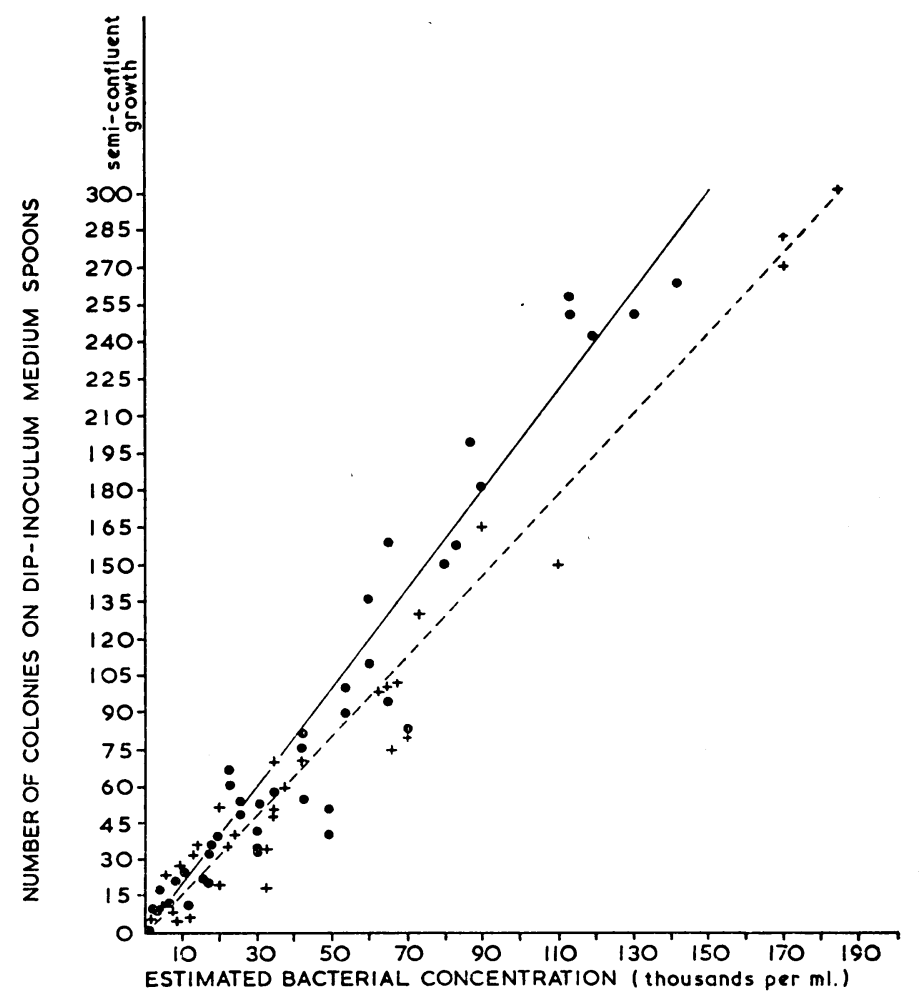

FIG. 2.-Estimated bacterial concentration in thousands per millilitre (standard methods). Gram-negative bacilli - Gram-positive cocci 


\section{Correlation between Spoon Colony Counts and Standard Viable Counts}

In order for the dip-inoculum spoon to be of value in routine urinary bacteriology it was necessary to show that the colony count obtainable on such a spoon is constant and in direct proportion to the original number of viable organisms present in the fresh urine. It was also necessary to show that the volume of urine retained on the spoon was small enough to avoid confluent growth when viable counts were below those expected from active infection. Accordingly, a series of comparisons were made between the number of colonies counted on the spoon after the dip-inoculum and the viable count obtained by standard methods.

Methods.-Tenfold dilutions were prepared, in sterile distilled water, from broth cultures of bacteria isolated from the urinary tract. From each dilution a dip-inoculum was made and $0.1 \mathrm{ml}$. spread over the surface of a standard E.D. agar plate. In addition, pour-plates were prepared with $0.1 \mathrm{ml}$. of each dilution. Later, when results showed no significant difference between the surface colony counts and the pour-plate counts, only the surface-colony-count method was employed. All the prepared cultures were incubated overnight at $37^{\circ} \mathrm{C}$.

Results.-Fig. 2 shows the correlation between the number of colonies obtained by the dip-inoculum and the calculated bacterial concentration per $\mathrm{ml}$. of fluid obtained by the standard technique. It will be seen that the sponn-colony counts show a direct proportion to the standard methods up to a concentration of $150,000 / \mathrm{ml}$., after which growth on the spoons tends to become confluent. It was expected that on spoons inoculated from fluid containing high bacterial concentrations a proportional reduction in the number of colonies would occur owing to the small surface area available. It was found, however, that only a reduction in colnny size occurred, without any significant reduction in numbers (Fig. 3). From these results the correlation factor between the spoon-colony counts and the standard viable counts can be calculated. For Gramnegative bacilli the factor by which the number of colonies on the spoon must be multiplied to give the viable count in thousands of bacteria per ml. is shown (Fig. 2) to be constant (up to near confluence) at 0.5 . For Gram-positive cocci the factor is seen to be also constant, but at 0.6. This slight factordifference is thought perhaps to be due to the short-chained or clumped organisms tending to produce relatively more

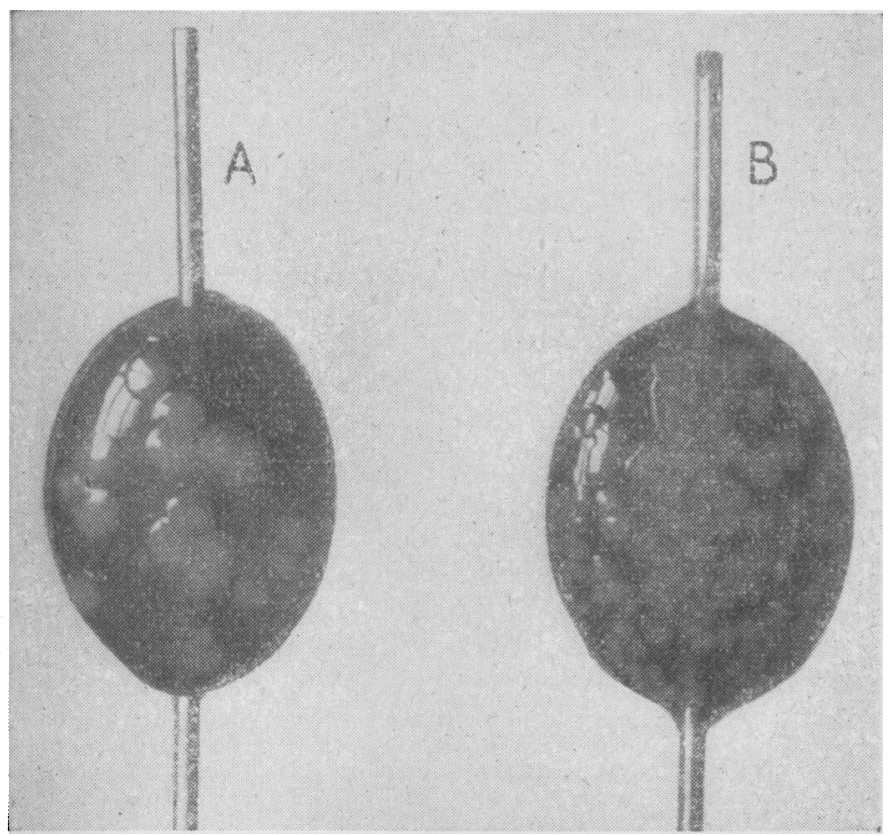

FIG. 3.-Medium spoons dip-inoculated from suspensions of Escherichia coli containing (A) 10,000 and (B) 100,000 organisms per ml., showing typical decrease in colony size with increasing concentracion. colonies on the plate because of the occasional break-up following the loop-spreading. In routine practice on urines, however, spoon-colony counts tend to be either fewer than 20 or confluent, depending on whether or not active infection is present.

\section{General-practitioner Diagnostic Outfit}

For routine laboratory use a postal outfit incorporating the dip-inoculum spoon is available. This consists of the described D.I.T.M. outfit and a plain 1-oz. (28-ml.) screw-capped universal bottle for the clean specimen of urine for microscopical and biochemical examination. An instruction sheet is included in each outfit. The whole is packed in a suitable cardboard box. It has been found that the D.I.T.M. outfit as described has a shelf life of at least six months.

In order to check that the performance of the medium spoon is not affected by postal delay batches of three spoons were inoculated from the same bacterial concentration. One spoon was incubated immediately; the second and third spoons were kept at room temperature for 24 and 48 hours respectively before incubation. No significant variation in the colony count was obtained. Also there was no effect on the colony count after storage at $4^{\circ} \mathrm{C}$. for 48 hours before incubation.

\section{Discussion}

The dip-inoculum technique described, though developed to meet the needs of the busy general practitioner in circumstances when the sample of urine cannot be dispatched to the laboratory without delay, has also been tried out with success in antenatal clinics. In these circumstances the speed and simplicity of use for the diagnosis of bacteriuria closely matches the Uristix dipsticks for the detection of protein and glucose.

In the clinical laboratory the method also has advantages over standard techniques in that naked-eye inspection of the bottles after overnight incubation would be all that is required to reach the correct diagnosis in respect of infection. Confirmation of bacterial identification and sensitivity tests on positive cases do, of course, require a subculture, but in all cases there is the advantage of speed, simplicity, and economy of effort.

Finally, it must be stressed that although polyurethane-coated metal spoons are suitable for the study of this technique and small-scale usage, disposable sterile plastic spoons would greatly facilitate the production and supply of large numbers of D.I.T.M. outfits.

\section{Summary}

A dip-inoculum transport-medium outfit is described which has been developed for the diagnosis of infections of the urinary tract by general practitioners who are unable to transport the specimen of urine to the laboratory without delay.

A standard inoculum of urine is obtained by dipping the medium spoon into the freshly passed mid-stream specimen. After inoculation the outfit may be returned by post to the laboratory, where a clinically accurate viable count can be assessed by inspection after overnight incubation.

We wish to thank the Medical Wire and Equipment Co. (Bath) Ltd., and in particular Mr. D. A. Turner, for their help in the production of the spoons used in this investigation, and $\mathrm{Mr}$. P. J. Basterfield for the photograph.

\section{REFERENCES}

Brumfitt, W., and Percival, A. (1964). F. clin. Path., 17, 482. Gallagher, D. J. A., Montgomerie, J. Z., and North, J. D. K. (1965). Brit. med. F., 1, 622.

Kass, E. H. (1956). Trans. Ass. Amer. Phycns, 69, 56.

Kass, (1960). Arch. intern. Med., 105, 194.

Leigh, D. A., and Williams, J. D. (1964). ‡. clin. Path., 17, 498.

Mond, N. C., Percival, A., Williams, J. D., and Brumfitt, W. (1965). Lancet, 1, 514.

Sandys, G. H. (1960). F. med. Lab. Teck., 17, 224. 